SCIENTIFIC REPORT

\title{
Sensitivity and specificity of scanning laser polarimetry using the GDx
}

\author{
S Munkwitz, J Funk, K U Loeffler, U Harbarth, S Kremmer
}

Br J Ophthalmol 2004;88:1142-1145. doi: 10.1136/bjo.2003.036756

Background/aims: To determine the sensitivity and the specificity of the GDx in the detection of (1) advanced glaucoma, (2) early glaucoma, and (3) nerve fibre bundle defects (NFBD).

Methods: Group A comprised 20 eyes with reproducible glaucomatous visual field defects confirmed by octopus perimetry, group B consisted of 10 eyes with normal visual fields but either glaucomatous NFBD or deterioration of the disc over time clearly visible upon flicker comparison, and group C included 16 eyes with glaucomatous or nonglaucomatous NFBD clearly visible on red free photographs. Forty four eyes of 22 healthy volunteers served as controls. The GDx printouts of all subjects were evaluated by three independent observers in a masked fashion and without the clinical picture of the optic disc. Two of the three observers (SK, UH) were GDx experts, one (KUL) was an untrained GDx user.

Results: Among the GDx experts, sensitivity/specificity was $100 \% / 100 \%$ (SK) and $90 \% / 100 \%$ (UH) in detecting advanced glaucoma, and 100\%/100\% (SK) and $90 \%$ / $100 \%(\mathrm{UH})$ in detecting early glaucoma. The sensitivity in detecting NFBD was only $37.5 \%$ (SK and UH). For the untrained GDx user the corresponding values were $50 \%$ / $100 \%$ (group A), 20\%/100\% (group B), and 12.5\%/91\% (group C).

Conclusion: Detection of (early) glaucoma damage by the GDx, evaluated by trained experts, can be extremely high. To optimise its benefit in clinical routine training in interpreting GDx printouts is highly recommended. Detection of localised NFBD is crucial, even for experts.

$\mathrm{T}$ he major threat from glaucoma is blindness, caused by progressive loss of retinal ganglion cell axons. This causes a decrease of the retinal nerve fibre layer (RNFL) thickness. ${ }^{1-4}$ Consequently the measurement of the decreasing RNFL thickness can provide important information for the early diagnosis and follow up of glaucoma. There is need for new diagnostic techniques, because it has been shown that by the time of clinical detection, $50 \%$ of the RNFL can already be lost. ${ }^{5}$

The GDx is a third generation nerve fibre analyser (NFA, Laser Diagnostic Technologies, San Diego, CA, USA), a scanning laser polarimeter that measures the phase shifting (retardation) of light that has double passed the birefringent fibres of the retinal nerve fibre layer (RNFL).

For the detection of glaucoma and for clinical usefulness, it is significant which stage of the disease can be detected by the device. Early diagnosis is very important for the treatment and prognosis of progressive neuropathies like glaucoma. Consequently our interest was focused on the results of the early glaucoma group. Improved diagnosis can help prevent unnecessary prophylactic treatment and so protect the patients from side effects of their therapy. It can also lower medical expenses.

The aim of this study is to evaluate the GDx in the detection of advanced glaucoma, early glaucoma, and nerve fibre bundle defects (NFBD) and to compare the data obtained with normal controls.

\section{METHODS}

\section{GDx}

The thickness of the peripapillary nerve fibre layer was determined using the GDx Nerve Fiber Analyzer (Software Version 2.0.10). This device uses a diode laser with a wavelength of $780 \mathrm{~nm}$ to create the polarised laser beam. ${ }^{6-9}$ The thickness data are transferred into a colour coded image. Thin areas of the RNFL relate to darker colours (blue-black), thicker areas to bright colours (yellow-orange). The operator has to position a blue ellipse around the margin of the optic disc. A second green ellipse with a given magnification factor of 1.75 disk diameters is displayed automatically peripherally to the blue centre ellipse. A so called TSNIT (temporal superior nasal inferior thickness) or double hump graph is calculated from 200 points along the green ellipse (fig 1).

The thickness image is divided into four segments: superior $120^{\circ}$ (for $0^{\circ}$ at a $12 \mathrm{o}^{\prime}$ clock position: $295^{\circ}-55^{\circ}$ ), nasal $70^{\circ}\left(55^{\circ}\right.$ $\left.125^{\circ}\right)$, inferior $120^{\circ}(125-245)$, and temporal $50^{\circ}\left(245^{\circ}-295^{\circ}\right)$. These segments are used to calculate the different parameters of the nerve fibre analysis.

The right and left eye of each patient and each volunteer was examined six times. The pupils were undilated. The quality of all images as determined by the GDx quality check was consistently $90 \%$ or higher. The best three images, where the optic disc was centred in such a way that it could be easily aligned, were chosen to create a mean image. All images were taken by the same operator (SM). He also positioned the ellipse around the margin of the optic disc.

\section{Patients}

Eighty three eyes were examined in this study. Forty four were eyes of healthy subjects and 39 were eyes of patients from the university eye clinic. The mean (standard deviation) age of the patients was 56.15 (SD 10.56) years and of the volunteers 23.81 (SD 1.54) years. The classification into the different groups was based on nerve fibre photography, visual field defects, flicker comparison of the optic disc, and intraocular pressure. Three groups of patients were defined.

Group A (advanced glaucoma) comprised 20 eyes with reproducible glaucomatous visual field defects confirmed by Octopus perimetry, group B (early glaucoma) consisted of 10 eyes with normal visual fields but either glaucomatous nerve fibre bundle defects or deterioration of the disc over time clearly visible upon flicker comparison, and group C (nerve

Abbreviations: NFBD, nerve fibre bundle defects; RNFL, retinal nerve fibre layer; SLP, scanning laser polarimetry. 

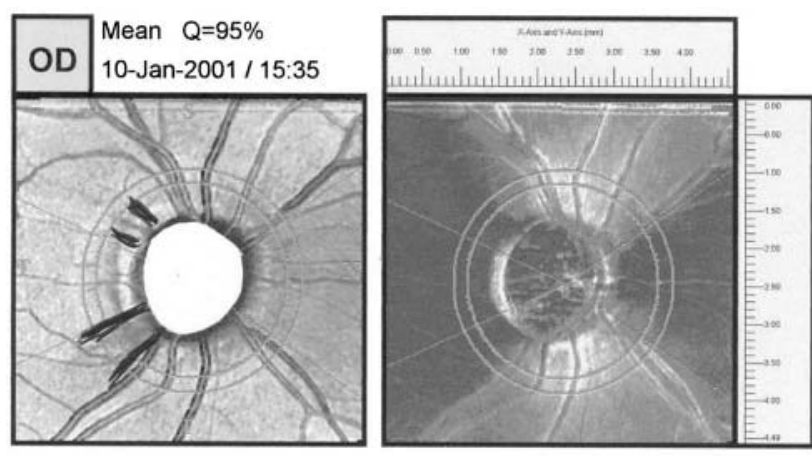

Nerve Fiber Layer

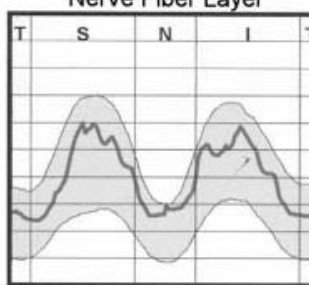

Nerve Fiber Layer

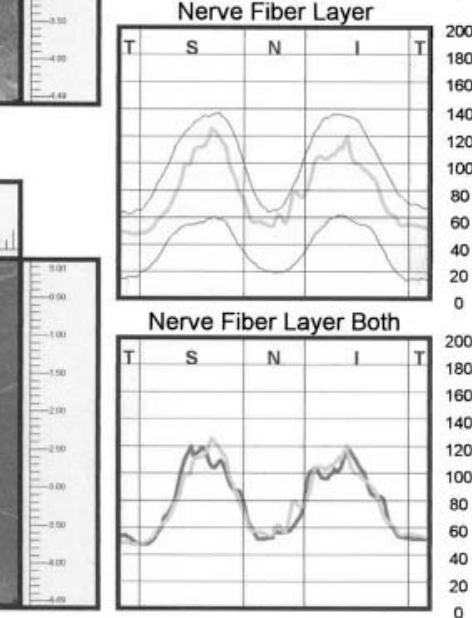

NERVE FIBER ANALYSIS
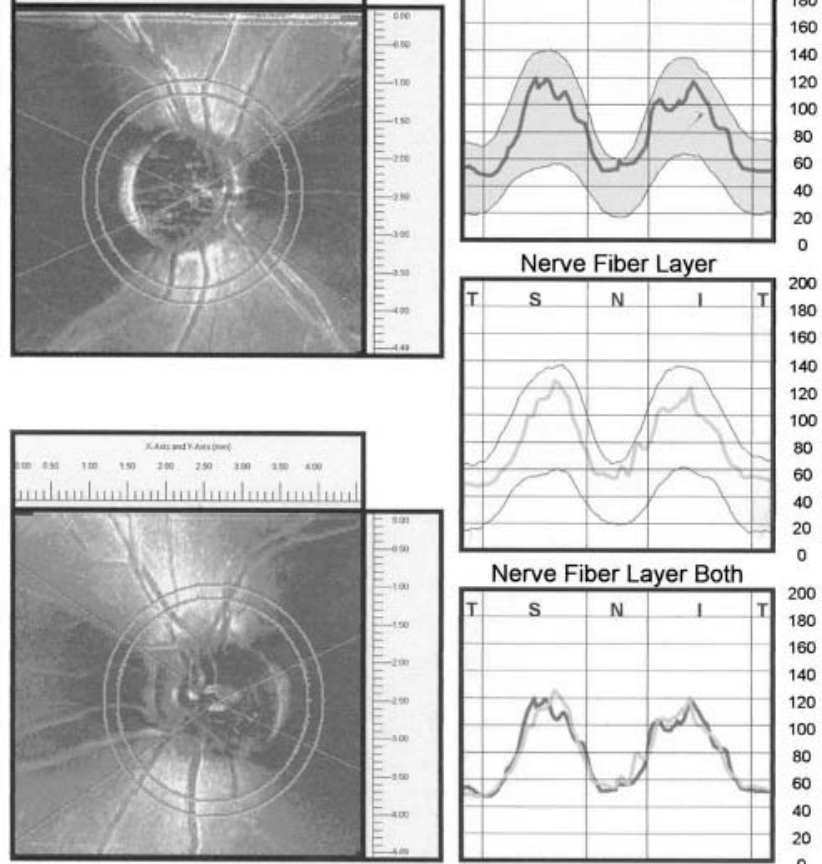

160

140

120

100
80
60

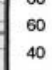

40
20
0

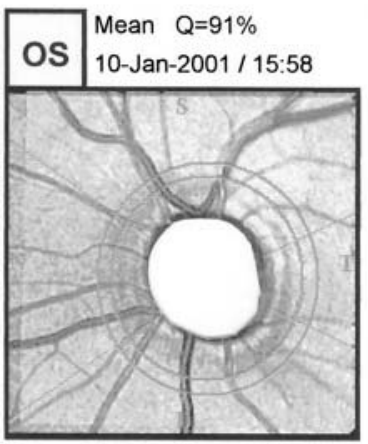

Figure 1 Example of a GDx-printout as it had to be evaluated by the three observers.

0

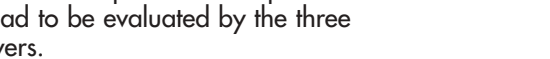

\begin{tabular}{|c|c|c|c|c|c|c|c|c|c|c|c|}
\hline & Aat value & Probatitily & Aet Value & Probability & Ditherences & & Act Value & Probabaiat & Ae value & Probabaily & Ditterence \\
\hline Symmetry & 1.04 & & 1.04 & & 0.00 & The Number & 28 & & 17 & & \\
\hline Superior Ratio & 1.85 & & 2.09 & & -0.24 & Ellipse Modulatan & 1.51 & & 1.62 & & -0.11 \\
\hline Intenor Raso & 1.78 & & 2.01 & & -0.24 & Average Thickness & 75 & & 75 & & 0 \\
\hline Superiontasal & 1.64 & $6.5 \mathrm{~K}$ & 1.76 & & -0.11 & Ellipse Average & 78 & & 78 & & 0 \\
\hline Max Modulation & 0.15 & 225 & 1.09 & & -0.24 & Superior Average & 89 & & 88 & & 1 \\
\hline Superiar Maximum & 95 & & 104 & & -10 & Interior Average & 89 & & 89 & & 0 \\
\hline Interior Maximum & 91 & & 100 & & -9 & Supenor integrat & 0.248 & & 0.245 & & 0.003 \\
\hline
\end{tabular}

1992-1990 Laser Diagnostic Technologies, inc, All Rights Reserved

9550 Waples Street, Suite \&1 105 San Diogo, CA 92121 (619)/558-9144 FAX 1619/558-914

fibre bundle defects) included 16 eyes with glaucomatous or non-glaucomatous nerve fibre bundle defects clearly visible on red free photographs (fig 2). Forty four eyes of 22 healthy volunteers served as controls. None of them had visual field defects or ocular diseases.

The GDx printouts of all patients and controls were evaluated by three independent observers in a masked

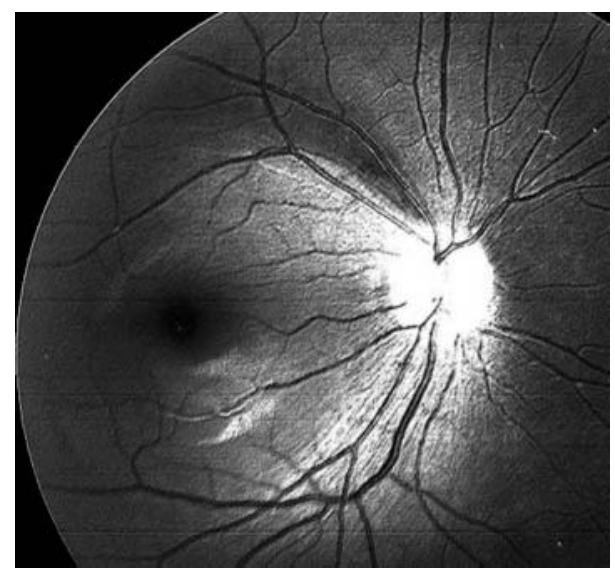

Figure 2 Example of a nerve fibre bundle defect clearly visible on red free photograph. fashion without any personal information (for example, age, sex, race, name) and without the clinical picture of the optic disc (fig 1), in order to base the decision on the RNFL only and to make detection more difficult. The observers had to decide via a questionnaire whether or not glaucoma and/or NFBD could be detected. If the observers could not make a diagnosis, they had the option to mark "not definable". The printouts of the patients and controls were in random order. Two of the three observers (SK, UH) were GDx experts. Observer SK has been working for more than nine years with scanning laser polarimetry and observer UH for more than 12 years. One (KUL) was an untrained, novice GDx user with only half a year's experience in using the GDx. This was to determine the influence of experience on the sensitivity and specificity of the GDx.

\section{RESULTS}

Expert l's results showed a high sensitivity and specificity of $100 \%$ among the advanced glaucoma and early glaucoma group. All of the 20 advanced and 10 early glaucomatous eyes were detected. All controls were defined as healthy. The specificity changed to $84.6 \%$, when the eight partner eyes from the glaucoma patients were included and presumed to be normal. These eyes showed no signs of glaucomatous damage when conventional diagnostic techniques (octopus perimetry, nerve fibre photography, and flicker comparison of the optic disc) were applied. 
The results of expert 2 in detecting glaucoma were similarly high: $90 \%$ sensitivity in detecting advanced and early glaucoma and $100 \%$ specificity, respectively. Eighteen of the 20 advanced and nine of the 10 early glaucomatous eyes were correctly classified, as well as all the healthy controls. On inclusion of the eight partner eyes, the specificity dropped to $86.5 \%$.

The corresponding values for the untrained GDx user were $50 \%$ sensitivity and $100 \%$ specificity for the advanced glaucoma. For the early glaucoma $20 \%$ sensitivity and $100 \%$ specificity were found. Ten of the 20 advanced and only two of the 10 early glaucomatous eyes could be detected. The eight partner eyes were mostly considered as not glaucomatous, so the specificity including these eyes was $96.2 \%$.

The sensitivity as far as the detection of NFBD was concerned was clearly worse. For experts 1 and 2 the sensitivity was $37.5 \%$. Only six of the 16 NFBD were identified. Furthermore expert 1 marked three eyes and expert 2 marked 14 eyes of the patients' group as "not definable". The untrained GDx user could only find two of the 16 NFBD clearly visible on red free photographs. This corresponds to a sensitivity of $12.5 \%$. Eleven eyes of the patients' group and three of the control group could not be defined.

\section{DISCUSSION}

This study evaluated the sensitivity and the specificity of the GDx in discriminating between advanced glaucoma, early glaucoma and normal controls, as well as its ability to detect NFBD.

Experts 1 and 2 show very good results with a 90\% and $100 \%$ sensitivity and a 100\% specificity in detecting early and advanced glaucoma. The high results achieved with the early glaucoma group are especially promising. Early diagnosis is the most decisive factor in reducing or preventing glaucomatous damage. It is interesting to note that most of the eight clinically healthy seeming partner eyes of the glaucoma group were considered glaucomatous by the experts. This could either indicate that the sensitivity of the GDx is higher than that of common diagnostic methods or show a high "false positive" result of the expert group.

Table 1 Glaucoma detection by the three observers (actual situation: 20 advanced glaucoma patients, 10 early glaucoma patients, 44 controls, and in parentheses the eight partner eyes without detectable glaucomatous disease from the glaucoma patients)

\begin{tabular}{|c|c|c|c|}
\hline$n=74(82)$ & $\begin{array}{l}\text { Advanced } \\
\text { glaucoma }\end{array}$ & $\begin{array}{l}\text { Early } \\
\text { glaucoma }\end{array}$ & No glaucoma \\
\hline \multicolumn{4}{|l|}{ Expert 1 (SK) } \\
\hline Advanced glaucoma & 20 & 0 & $0(8)$ \\
\hline Early glaucoma & 0 & 10 & $0(0)$ \\
\hline No glaucoma & 0 & 0 & $44(44)$ \\
\hline Sensitivity & $100 \%$ & $100 \%$ & \\
\hline Specificity & & & $100 \%(84.6 \%)$ \\
\hline \multicolumn{4}{|l|}{ Expert $2(\mathrm{UH})^{*}$} \\
\hline Advanced glaucoma & 18 & 0 & $0(7)$ \\
\hline Early glaucoma & 0 & 9 & $0(0)$ \\
\hline No glaucoma & 2 & 1 & $44(45)$ \\
\hline Sensitivity & $90 \%$ & $90 \%$ & \\
\hline Specificity & & & $100 \%(86.5 \%)$ \\
\hline \multicolumn{4}{|c|}{ Untrained GDx user (KUL) } \\
\hline Advanced glaucoma & 10 & 0 & $0(2)$ \\
\hline Early glaucoma & 0 & 2 & $0(0)$ \\
\hline No glaucoma & 10 & 8 & $44(50)$ \\
\hline Sensitivity & $50 \%$ & $20 \%$ & \\
\hline Specificity & & & $100 \%(96.2 \%)$ \\
\hline
\end{tabular}

Table 2 NFBD detection by the three observers (actual situation: 16 eyes with NFBD, 44 controls, and in parentheses 23 eyes from the glaucoma patients without detectable NFBD on red free photography)

\begin{tabular}{lcc}
\hline $\mathbf{n}=60$ (83) & NFBD: yes & NFBD: no \\
\hline Expert 1 (SK) & 6 & $0(5)$ \\
NFBD: yes & 10 & $44(62)$ \\
NFBD: no & $37.5 \%$ & $100 \%(92.5 \%)$ \\
Sensitivity & & \\
Specificity & & $0(8)$ \\
Expert 2 (UH) & 6 & $44(59)$ \\
NFBD: yes & 10 & $100 \%(88.1 \%)$ \\
NFBD: no & $37.5 \%$ & $4(8)$ \\
Sensitivity & & $40(59)$ \\
Specificity & 2 & $90.9 \%(88.1 \%)$ \\
Untrained GDx user (KUL) & \\
NFBD: yes & 14 & \\
NFBD: no & $12.5 \%$ & \\
Sensitivity & & \\
Specificity & &
\end{tabular}

More sensitive procedures to detect deterioration over time could help to find a better and more individual therapy for ocular hypertension and early glaucoma patients. It would also allow a better prediction of the moment when they are going to convert into an advanced glaucomatous stage. Follow up studies and further clinical experience with the GDx will be necessary to find out whether it fulfils these requirements.

The results of the untrained clinical user in detecting early and advanced glaucoma were clearly worse, with $20 \%$ and $50 \%$ sensitivity.

The detection of NFBD, identified clearly on red free photographs, was very difficult for all three observers. The sensitivities of $37.5 \%$ (six of 16 NFBD, both experts) and $12.5 \%$ (two of 16 NFBD, untrained clinical user) show that the GDx is not suitable for the detection of localised NFBD.

Several studies have been published to determine the sensitivity and the specificity of scanning laser polarimetry (SLP). ${ }^{10-14}$ It is difficult to compare them because they used different parameters for analysis, different versions of the NFA/GDx, and different stages of glaucoma damage. One of the first studies about SLP was made with the NFA I, a predecessor of the GDx. ${ }^{11}$ The authors found a sensitivity of $96 \%$ and a specificity of $93 \%$. There were no early glaucoma patients included in the study. All the glaucoma patients had typical glaucomatous visual field defects and were scored "outside normal limits" on the glaucoma hemifield test. The calculation of the sensitivity and the specificity was performed by an objective analysis of different parameters and cut off values. Some studies also evaluated the GDx printouts in a subjective manner by using trained experts. ${ }^{15-17}$ The results of the expert group in our study were better than the sensitivity and specificity reported in these studies.

Nicolela et $a l^{16}$ discriminated between three different groups of glaucoma patients (advanced, localised, mixed group) and normal controls. All three groups showed visual field defects. By means of subjective evaluation 93\%, 70\%, and $47 \%$ of the glaucomas from the advanced, localised, and mixed groups respectively were found, and $97 \%$ of the controls were correctly identified. The best objective discrimination (different combinations of GDx parameters) identified the localised and mixed glaucoma group better, but showed a specificity of only $69 \%$. The high degree of sensitivity by our two experts in the detection of early glaucoma confirms that the GDx is highly sensitive even for early to moderate glaucoma. Colen et $a l^{18}$ showed that a subjective clinical judgment leads to better discrimination 
than the number as a standard parameter of the GDx, especially in the group with mild glaucoma.

Our study confirms that a subjective evaluation of GDx measurements performed by experienced observers can show very good results. The mean age of the control group (23.81 (SD 1.54) years) was significantly lower than the age of the patients' group (56.15 (SD 10.56) years). It is possible that the higher RNFL measurements of the control group led to the assumption that the subject was healthy and this might have positively biased the specificity. On the other hand the great interindividual variability of the RNFL that has been reported in histological measurements ${ }^{19}$ and the large overlap in RNFL measurements with SLP between normal and glaucomatous eyes ${ }^{20}$ might neutralise this effect.

In other studies, one eye per subject is often randomly selected; this was not the case in our study. The comparison of both eyes (asymmetries) could have facilitated the decision of the observers. However, the fact that the age and the optic disc were masked made it more difficult to make a diagnosis.

The GDx can be a valuable additional tool in detecting glaucoma damage even at an early stage. Sensitivity and specificity in interpreting the GDx measurements can be extremely high when the evaluation is performed by trained experts, but among untrained clinical users sensitivity is poor. From these findings we conclude that training in interpreting GDx printouts can greatly improve the sensitivity and is highly recommended in order to optimise its benefits in clinical routine. However, for the detection of localised nerve fibre bundle defects the GDx is not suitable.

\section{Authors' affiliations}

S Munkwitz, J Funk, Department of Ophthalmology, Albert-LudwigsUniversity, Freiburg, Germany

K U Loeffler, Department of Ophthalmology, University of Bonn, Bonn, Germany

U Harbarth, Laser Diagnostic Technologies (LDT) Europe, Schriesheim, Germany

S Kremmer, Department of Ophthalmology, University of Essen, Essen, Germany

Correspondence to: Professor Dr J Funk, Albert-Ludwigs-University, Killianstr. 5, Freiburg 79106, Germany; funk@aug.ukl.uni-freiburg.de

Accepted 1 February 2004

\section{REFERENCES}

1 Airaksinen PJ, Drance SM. Neuroretinal rim area and retinal nerve fiber layer in glaucoma. Arch Ophthalmol 1985;103:203-4.

2 Hoyt WF, Newman NM. The earliest observable defect in glaucoma? Lancet 1972;1:692-3.

3 Quigley HA, Miller NR, George T. Clinical evaluation of nerve fiber layer atrophy as an indicator of glaucomatous optic nerve damage. Arch Ophthalmol 1980;98:1564-71.

4 Tuulonen A, Airaksinen PJ. Initial glaucomatous optic disk and retinal nerve fiber layer abnormalities and their progression. Am J Ophthalmol 1991;111:485-90.

5 Quigley HA, Addicks EM. Quantitative studies of retinal nerve fiber layer defects. Arch Ophthalmol 1982;100:807-14.

6 Dreher AW, Reiter K, Weinreb RN. Spatially resolved birefringence of the retinal nerve fibre layer assessed with a retinal laser ellipsometer. Appl Opt 1992;31:3730-5.

7 Dreher AW, Reiter K, Weinreb RN. Measurement of the circumpapillary nerve fiber layer distribution by polarimetry. Invest Opthalmol Vis Sci 1991;32:811.

8 Dreher AW, Reiter K. Retinal laser ellipsometry: a new method for measuring the retinal nerve fiber layer thickness distribution? Clin Vision Sci 1992;7:481-8.

9 Weinreb RN, Dreher AW, Coleman A, et al. Histopathologic validation of Fourier-ellipsometry measurements of retinal nerve fiber layer thickness. Arch Ophthalmol 1990; 108:557-60.

10 Paczka JA, Friedman DS, Quigley HA, et al. Diagnostic capabilities of frequency-doubling technology, scanning laser polarimetry, and nerve fiber layer photographs to distinguish glaucomatous damage. Am J Ophthalmol 2001;131:188-97.

11 Tjon-Fo-sang MJ, Lemij HG. The sensitivity and specificity of nerve fiber layer measurements in glaucoma as determined with scanning laser polarimetry. Am J Ophthalmol 1997; 123:62-9.

12 Vitale S, Smith TD, Quigley T, et al. Screening performance of functional and structural measurements of neural damage in open-angle glaucoma: a case-control study from the Baltimore Eye Survey. J Glaucoma 2000;9:346-56.

13 Weinreb RN, Zangwill L, Berry CC, et al. Detection of glaucoma with scanning laser polarimetry. Arch Ophthalmol 1998;116:1583-9.

14 Fabre K, Michiels I, Zeyen T. The sensitivity and specificity of TOP, FDP and GDX in screening for early glaucoma. Bull Soc Belge Ophtalmol 2000;275:17-23.

15 Choplin NT, Lundy DC. The sensitivity and specificity of scanning laser polarimetry in the detection of glaucoma in a clinical setting. Ophthalmology 2001; 108:899-904.

16 Nicolela MT, Martinez-Bello C, Morrison CA, et al. Scanning laser polarimetry in a selected group of patients with glaucoma and normal controls. Am J Ophthalmol 2001; 132:845-54.

17 Sanchez-Galeana C, Bowd C, Blumenthal EZ, et al. Using optical imaging summary data to detect glaucoma. Ophthalmology 2001;108:1812-18.

18 Colen TP, Lemii HG. Sensitivity and specificity of the GDx: clinical judgment of standard printouts versus the number. J Glaucoma 2003;12:129-33.

19 Jonas JB, Schmidt AM, Muller-Bergh JA, et al. Human optic nerve fiber count and optic disc size. Invest Ophthalmol Vis Sci 1992;33:2012-18.

20 Weinreb RN, Shakiba S, Zangwill L. Scanning laser polarimetry to measure the nerve fiber layer of normal and glaucomatous eyes. Am J Ophthalmol $1995 ; 119: 627-36$. 